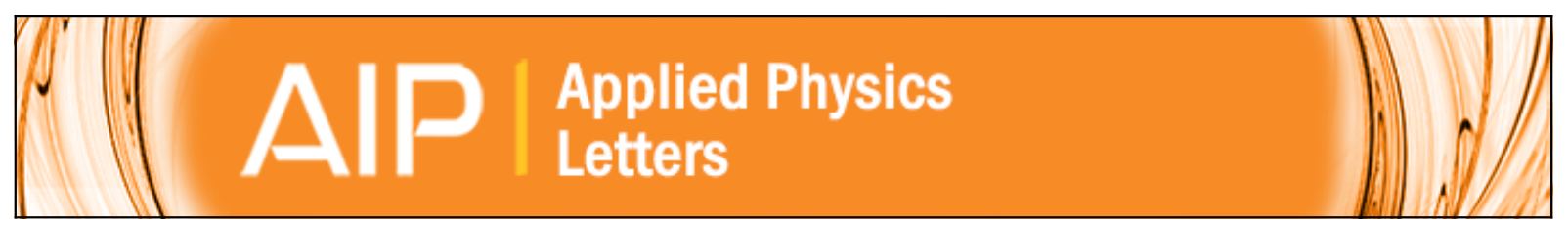

\title{
Effect of $\mathrm{NiO}$ inserted layer on spin-Hall magnetoresistance in $\mathrm{Pt} / \mathrm{NiO} / \mathrm{YIG}$ heterostructures
}

T. Shang, Q. F. Zhan, H. L. Yang, Z. H. Zuo, Y. L. Xie, L. P. Liu, S. L. Zhang, Y. Zhang, H. H. Li, B. M. Wang, Y. H. Wu, S. Zhang, and Run-Wei Li

Citation: Applied Physics Letters 109, 032410 (2016); doi: 10.1063/1.4959573

View online: http://dx.doi.org/10.1063/1.4959573

View Table of Contents: http://scitation.aip.org/content/aip/journal/apl/109/3?ver=pdfcov

Published by the AIP Publishing

\section{Articles you may be interested in}

Effect of IrMn inserted layer on anomalous-Hall resistance and spin-Hall magnetoresistance in Pt/lrMn/YIG heterostructures

J. Appl. Phys. 120, 133901 (2016); 10.1063/1.4964114

Isothermal tuning of magnetic coercivity in $\mathrm{NiFe} / \mathrm{NiO} /[\mathrm{Co} / \mathrm{Pt}]$ heterostructures with orthogonal easy axes J. Appl. Phys. 118, 093901 (2015); 10.1063/1.4929760

Anti-damping spin transfer torque through epitaxial nickel oxide Appl. Phys. Lett. 106, 162406 (2015); 10.1063/1.4918990

Exchange magnetic field torques in YIG/Pt bilayers observed by the spin-Hall magnetoresistance Appl. Phys. Lett. 103, 032401 (2013); 10.1063/1.4813760

Polarity of anomalous Hall effect hysteresis loops in [ Pt/Co ] 15/AF/[ Co/Pt ] $15(\mathrm{AF}=\mathrm{Fe} \mathrm{Mn}, \mathrm{NiO})$ multilayers with perpendicular anisotropy

J. Appl. Phys. 97, 013901 (2005); 10.1063/1.1825628

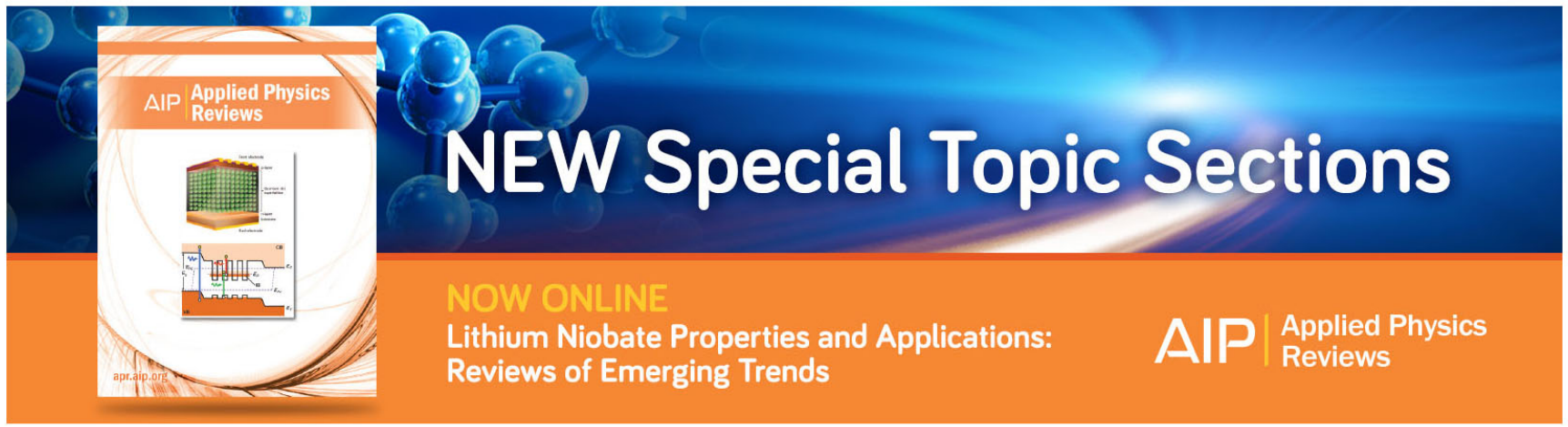




\title{
Effect of NiO inserted layer on spin-Hall magnetoresistance in Pt/NiO/YIG heterostructures
}

\author{
T. Shang, ${ }^{1, a)}$ Q. F. Zhan, ${ }^{1, b)}$ H. L. Yang, ${ }^{1}$ Z. H. Zuo, ${ }^{1}$ Y. L. Xie,,${ }^{1}$ L. P. Liu, ${ }^{1}$ S. L. Zhang, ${ }^{1}$ \\ Y. Zhang, ${ }^{1}$ H. H. Li, ${ }^{1}$ B. M. Wang, ${ }^{1}$ Y. H. Wu, ${ }^{2}$ S. Zhang, ${ }^{3, c)}$ and Run-Wei Li $\left.{ }^{1, d}\right)$ \\ ${ }^{1}$ Key Laboratory of Magnetic Materials and Devices and Zhejiang Province Key Laboratory of Magnetic \\ Materials and Application Technology, Ningbo Institute of Material Technology and Engineering, \\ Chinese Academy of Sciences, Ningbo, Zhejiang 315201, China \\ ${ }^{2}$ Department of Electrical and Computer Engineering, National University of Singapore, 4 Engineering Drive 3, \\ Singapore 117583 \\ ${ }^{3}$ Department of Physics, University of Arizona, Tucson, Arizona 85721, USA
}

(Received 24 April 2016; accepted 12 July 2016; published online 22 July 2016)

\begin{abstract}
We investigate spin-current transport with an antiferromagnetic insulator $\mathrm{NiO}$ thin layer by means of the spin-Hall magnetoresistance (SMR) over a wide range of temperature in $\mathrm{Pt} / \mathrm{NiO} / \mathrm{Y}_{3} \mathrm{Fe}_{5} \mathrm{O}_{12}$ ( $\mathrm{Pt} / \mathrm{NiO} / \mathrm{YIG}$ ) heterostructures. The $\mathrm{SMR}$ signal is comparable to that without the NiO layer as long as the temperature is near or above the blocking temperature of the $\mathrm{NiO}$, indicating that the magnetic fluctuation of the insulating $\mathrm{NiO}$ is essential for transmitting the spin current from the $\mathrm{Pt}$ to YIG layer. On the other hand, the SMR signal becomes negligibly small at low temperature, and both conventional anisotropic magnetoresistance and the anomalous Hall resistance are extremely small at any temperature, implying that the insertion of the $\mathrm{NiO}$ has completely suppressed the $\mathrm{Pt}$ magnetization induced by the YIG magnetic proximity effect (MPE). The dual roles of the thin $\mathrm{NiO}$ layer are, to suppress the magnetic interaction or MPE between Pt and YIG, and to maintain efficient spin current transmission at high temperature. Published by AIP Publishing.

[http://dx.doi.org/10.1063/1.4959573]
\end{abstract}

Spin current, the motion of spin angular momentum, has attracted intense interest due to the prospects of low-energyconsumption spintronic devices. ${ }^{1,2}$ Several experimental techniques have been developed to generate and manipulate the spin current, e.g., spin pumping, ${ }^{3-5}$ spin Seebeck effect, ${ }^{6-8}$ and spin-Hall effect (SHE). ${ }^{9-11}$ Recently, the generation and propagation of spin current in antiferromagnets (AFMs) have been extensively investigated by various techniques. ${ }^{12-22}$ Especially, the thermally injected or dynamically pumped spin current from ferromagnetic (FM) $\mathrm{Y}_{3} \mathrm{Fe}_{5} \mathrm{O}_{12}$ (YIG) layer can flow into the $\mathrm{NiO}$ or $\mathrm{CoO}$ antiferromagnetic insulator (AFMI) layer and reach the Pt or Ta nonmagnetic metal (NM) layer where it can be converted into charge current by means of the inverse spin-Hall effect (ISHE). ${ }^{18-22}$ By introducing a thin AFMI layer, the ISHE voltage is largely enhanced and exhibits a nonmonotonic temperature or an AFMI thickness dependence, with a maximum value appearing near the antiferromagnetic (AFM) ordering temperature of AFMI or at the AFMI thickness of $\sim 1-2 \mathrm{~nm}$, respectively. ${ }^{18-22}$

Several theoretical models have been proposed for the propagation of injected spin current through AFMs in NM/ AFM/FM heterostructures. ${ }^{23-26}$ These models describe the spin-current transport and its enhancement by assuming that the AFMs are ordered at room temperatures, while the ordering temperatures of thin AFM films are well below the room temperature. ${ }^{27}$ In most of these experimental or theoretical

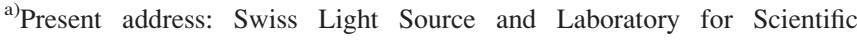
Developments and Novel Materials, Paul Scherrer Institute, CH-5232 Villigen PSI, Switzerland

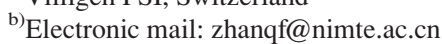

${ }^{c)}$ Electronic mail: zhangshu@email.arizona.edu

d)Electronic mail: runweili@nimte.ac.cn
}

investigations, the spin current carried by spin waves is produced in YIG layer and flows into the AFMI layer. ${ }^{18-26}$ In this paper, we provide an alternative method of spin current source, namely, the spin current is generated in the Pt layer via $\mathrm{SHE}^{9-11}$ and the YIG layer serves merely as a spin current absorber. Specifically, we study the magnetoresistance in $\mathrm{Pt} / \mathrm{NiO} / \mathrm{YIG}$ heterostructures in which the spin-Hall magnetoresistance (SMR) has been correlated with the spin current absorbed by the YIG layer.

The $\mathrm{Pt} / \mathrm{NiO} / \mathrm{YIG}$ heterostructures were prepared on (111)-orientated $\mathrm{Gd}_{3} \mathrm{Ga}_{5} \mathrm{O}_{12}$ (GGG) substrates in a combined ultra-high vacuum $\left(10^{-9}\right.$ Torr) pulse laser deposition (PLD) and sputter system. The high-quality $\mathrm{YIG}$ and $\mathrm{NiO}$ layers were deposited via PLD technique. The deposition temperature and oxygen pressure were kept at $750^{\circ} \mathrm{C}$ and 80 mTorr for $\mathrm{YIG}$ growth, and at $600^{\circ} \mathrm{C}$ and $50 \mathrm{mTorr}$ for $\mathrm{NiO}$ growth, respectively. The energy density and the frequency of laser are $4 \mathrm{~J} / \mathrm{cm}^{2}$ and $2 \mathrm{~Hz}$, respectively. The distance between target and substrate is around $55 \mathrm{~mm}$. After growth, the samples were annealed under the depositing conditions for $1 \mathrm{~h}$ to ensure a complete and homogeneous oxygenation. The top $\mathrm{Pt}$ films were sputtered in an in situ process in a 4 mTorr argon atmosphere at room temperature. In this study, the thicknesses of YIG and Pt layers are fixed at 60 and $3 \mathrm{~nm}$, respectively, while the $\mathrm{NiO}$ thickness ranges from 0 to $8 \mathrm{~nm}$. Figure 1(a) plots a representative $\mathrm{X}$-ray diffraction (XRD) pattern for an epitaxial YIG film near the (444) reflection. Clear Laue oscillations indicate an ideal flatness and uniformity of the prepared film. As shown in Fig. 1(b), no indication of impurities or misorientation was detected in the range of $20^{\circ}-80^{\circ}$. The atomic force microscope surface topography of $\mathrm{Pt} / \mathrm{NiO}(3) /$ YIG heterostructure over an area of $3 \mu \mathrm{m} \times 3 \mu \mathrm{m}$ in Fig. 1(c) 

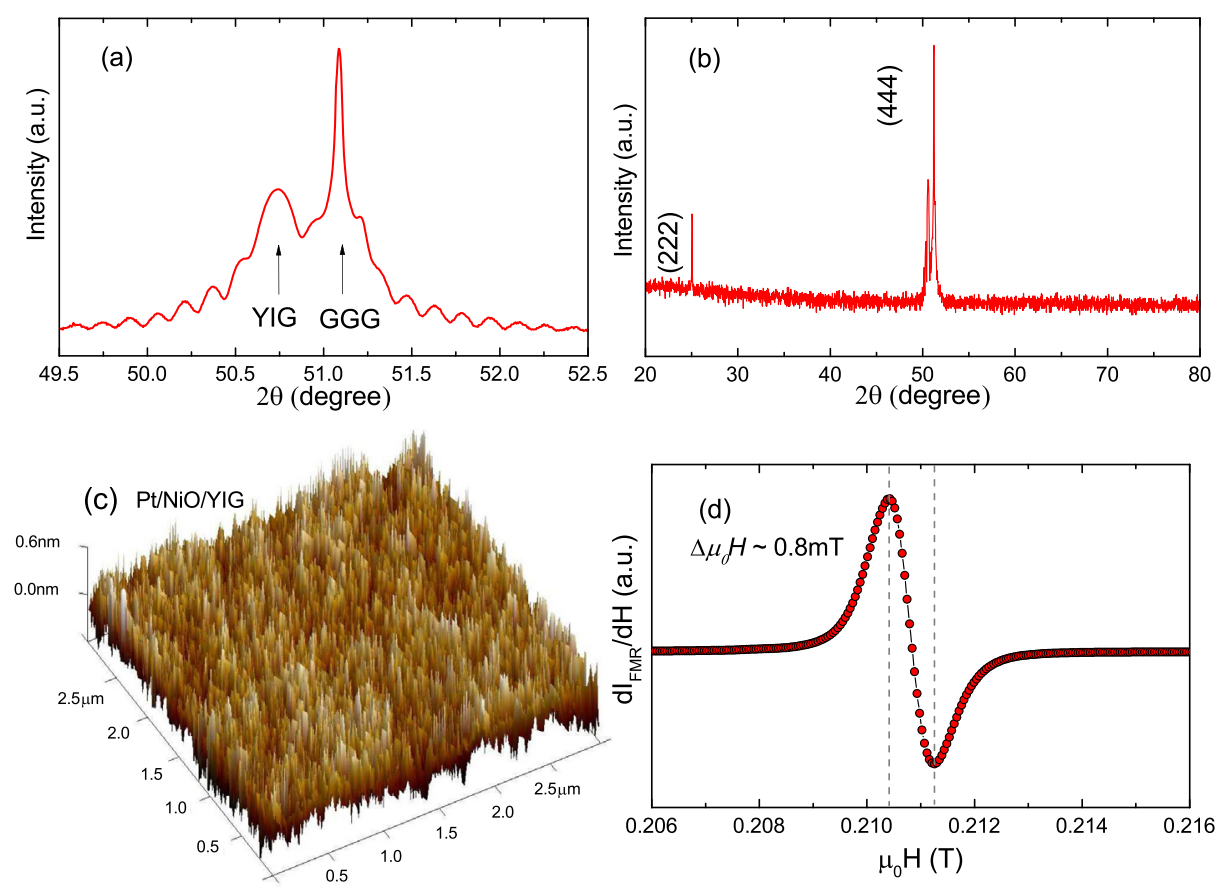

FIG. 1. The representative 20- $\omega$ XRD patterns for a YIG/GGG film (a) near the (444) reflections and (b) from $20^{\circ}$ to $80^{\circ}$. (c) Three dimensional plot of the atomic force microscope surface topography for $\mathrm{Pt} / \mathrm{NiO}(3) / \mathrm{YIG}$ over an area of $3 \mu \mathrm{m} \times 3 \mu \mathrm{m}$. (d) A representative FMR derivative absorption spectrum of YIG film. reveals a root-mean-square surface roughness of $0.14 \mathrm{~nm}$ (the number in the brackets shows the thickness of $\mathrm{NiO}$ layer in $\mathrm{nm}$ ), indicating that the prepared films are atomically flat. A representative ferromagnetic resonance (FMR) derivative absorption spectrum of YIG films shown in Fig. 1(d) exhibits a peak-to-peak value $\Delta \mu_{0} \mathrm{H}$ of $0.8 \mathrm{mT}$, which was measured at a radio frequency of $9.39 \mathrm{GHz}$ and a power of $0.1 \mathrm{~mW}$ with an in-plane magnetic field at room temperature. The estimated damping constant $\alpha=2.1 \times 10^{-3}$ is comparable with the previously reported value. ${ }^{28}$ For a reference, $\mathrm{Pt} / \mathrm{NiO} /$ $\mathrm{MgO}(001)$ heterostructures were additionally prepared by using the above parameters.

As shown in the right panel of Fig. 2, all the films were patterned into a Hall-bar geometry (central area: $0.3 \mathrm{~mm}$ $\times 10 \mathrm{~mm}$; electrodes: $0.3 \mathrm{~mm} \times 1 \mathrm{~mm}$ ) by using a shadow mask during the growth. The anisotropic magnetoresistance (AMR) were measured in a magnetic field of $2 \mathrm{~T}$ for both
$\mathrm{Pt} / \mathrm{NiO} / \mathrm{YIG}$ and $\mathrm{Pt} / \mathrm{NiO} / \mathrm{MgO}$ heterostructures. The absence of $\mathrm{AMR}$ in $\mathrm{Pt} / \mathrm{NiO} / \mathrm{MgO}$ implies that the $\mathrm{NiO}$ moments cannot be polarized by such magnetic field. Both the Pt/YIG and $\mathrm{Pt} / \mathrm{NiO}(1) / \mathrm{YIG}$ heterostructures demonstrate a clear SMR with the amplitudes reaching $6.1 \times 10^{-4}$ and $4.5 \times 10^{-4}$ at room temperature, respectively [see Figs. 2(c) and 2(f)], implying that the spin current generated by SHE in Pt can transport through $\mathrm{NiO}$ and interact with YIG. Apparently, the spin current can pass through $\mathrm{NiO}$ from both directions, i.e., $\mathrm{Pt} \rightarrow \mathrm{NiO} \rightarrow \mathrm{YIG}$ or $\mathrm{YIG} \rightarrow \mathrm{NiO} \rightarrow \mathrm{Pt}^{18,19,22}$ For Pt/ YIG, as shown in Fig. 2(b), a conventional AMR (CAMR) induced by the magnetic proximity effect (MPE) always coexists with the SMR and its maximum amplitude of $2.2 \times 10^{-4}$ is comparable to the SMR. However, for $\mathrm{Pt} /$ $\mathrm{NiO}(1) / \mathrm{YIG}$, there is no clear CAMR at any temperatures [see Fig. 2(e)], and the observed AMR in $x y$ plane $\left(\theta_{x y}\right)$ is entirely from the SMR. As shown in Figs. 2(d) and 2(f), the
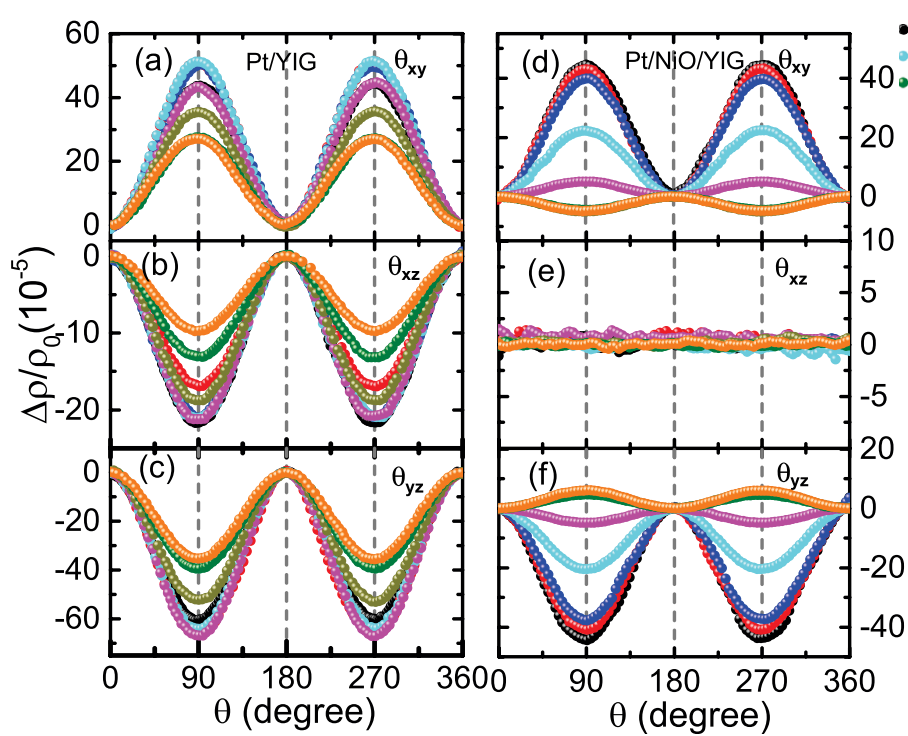

$300 \mathrm{~K} \cdot 250 \cdot 200$

$150 \cdot 100 \cdot 50$

$10 \cdot 3 \uparrow z$

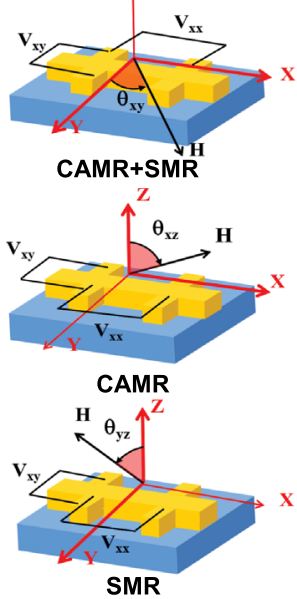

FIG. 2. The AMR at various temperatures for (a)-(c) Pt/YIG and (d)-(f) Pt/ $\mathrm{NiO}(1) / Y I G$ with magnetic field varied within the $x y, x z$, and $y z$ planes. The right panel shows the schematic plots of the longitudinal and transverse resistance measurements. The magnetic field orientations $\theta_{x y}, \theta_{x z}$, and $\theta_{y z}$ are with respect to the $y$-, $z$-, and $z$-axes, respectively. The electric current is applied along the $x$-axis. 


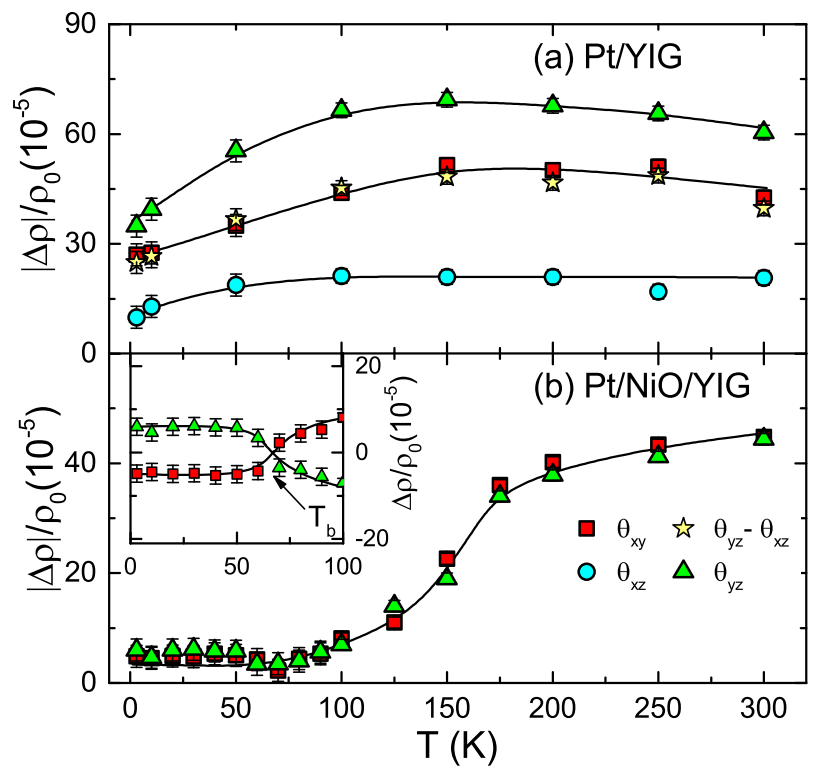

FIG. 3. Temperature dependence of AMR amplitude for (a) Pt/YIG and (b) $\mathrm{Pt} / \mathrm{NiO}(1) / \mathrm{YIG}$ heterostructures. The cubic, circle, and triangle symbols stand for the $\theta_{x y}, \theta_{x z}$, and $\theta_{y z}$ scans, respectively. The stars represent the difference between the SMR and the CAMR $\left(\theta_{y z}-\theta_{x z}\right)$. The inset of (b) shows the $\Delta \rho / \rho_{0}$ below $100 \mathrm{~K}$ for $\mathrm{Pt} / \mathrm{NiO}(1) / \mathrm{YIG}$. The solid lines are guides to the eye.

$\theta_{x y}$ and $\theta_{y z}$ scans exhibit a similar behavior, reinforce the notion that the MPE on Pt is completely destroyed by the $\mathrm{NiO}$ layer. Further support on the absence of MPE is provided by the anomalous Hall resistance (AHR). For instance, the AHR at $7 \mathrm{~T}$ for $\mathrm{Pt} / \mathrm{NiO}(1) / \mathrm{YIG}$ is $4.6 \mathrm{~m} \Omega$ at $5 \mathrm{~K}$, which is 6 times smaller than the value of $27.6 \mathrm{~m} \Omega$ for Pt/YIG. For $t_{\mathrm{NiO}} \geq 3 \mathrm{~nm}$, the AHR becomes undetectable. Compared to the $\mathrm{Pt} / \mathrm{IrMn} / \mathrm{YIG}, \mathrm{Pt} / \mathrm{Cu} / \mathrm{YIG}$, or Pt/Au/YIG heterostructures, only $26 \%$ of the SMR is lost by the insertion of $1 \mathrm{~nm} \mathrm{NiO}$ layer, while over $80 \%$ of the SMR is suppressed by the insertion of $1 \mathrm{~nm} \mathrm{IrMn}, \mathrm{Cu}$, or Au layer at room temperature. ${ }^{29-31}$ Our experiments illustrate that the $\mathrm{NiO}$ inserted layer could efficiently block the MPE while still allow the transport of most of the spin current at room temperature.

All the AMR amplitudes are summarized in Fig. 3 as a function of temperature. For Pt/YIG, the SMR exhibits a nonmonotonic temperature dependence and acquires its maximum value of $6.9 \times 10^{-4}$ around $150 \mathrm{~K}$. While for $\theta_{x y}$ scan in which both CAMR and SMR contribute to the total AMR, the amplitude is almost identical to the difference between SMR and CAMR [see stars in Fig. 3(a)], i.e., $|\Delta \rho| / \rho_{0}\left(\theta_{x y}\right)$ $=|\Delta \rho| / \rho_{0}\left(\theta_{y z}\right)-|\Delta \rho| / \rho_{0}\left(\theta_{x z}\right)$. After inserting a $\mathrm{NiO}$ layer, as shown in Fig. 3(b), $|\Delta \rho| / \rho_{0}\left(\theta_{x y}\right)=|\Delta \rho| / \rho_{0}\left(\theta_{y z}\right)$, which indicates that the SMR dominates the AMR for $\theta_{x y}$ scan due to the absence of MPE. Similar results have been previously reported in MPE-free Rh/YIG bilayers. ${ }^{32}$

The temperature dependence of the SMR is highly nontrivial. The magnitude of the SMR decreases sharply when the temperature becomes lower than the blocking temperature. Qualitatively, one may attribute the decrease to the reduction of the number of AFM magnons ${ }^{22}$ since the spin current carriers in the $\mathrm{NiO}$ layer magnons whose population rapidly decreases as temperature decreases. What is more interesting is the SMR changes sign around $70 \mathrm{~K}$, and then the SMR stays at a constant value below $70 \mathrm{~K}$, about 10 times smaller than that of room temperature. The magnetic characterization for the exchange bias field suggests that the $\mathrm{NiO}$ moments in $\mathrm{Pt} / \mathrm{NiO}(1) / \mathrm{YIG}$ become antiferromagnetcially ordered when approaching the blocking temperature $T_{\mathrm{b}} \sim 70 \mathrm{~K}$ (see details in Fig. 4). At present, it is unclear what governs the low temperature SMR behavior; it appears to be correlated with the formation of the AFM ordering.

The AFM ordering temperature for a very thin AFM film is expected to be well below the corresponding Néel temperature. ${ }^{27}$ It is rather difficult to measure the ordering temperature on the ultrathin AFM film. One approximate way is to study temperature dependence of exchange bias field $H_{\mathrm{e}}$ in which the blocking temperature is defined as the $H_{\mathrm{e}}$ reduces to zero. However, the ordering temperature could be quite different from the blocking temperature since the former characterizes the long range exchange correlation while the latter is simple description of the AFM anisotropy constant. Nevertheless, we present the exchange bias data to approximately estimate the ordering temperature. Figure 4(a) shows the typical hysteresis loops at various temperatures for $\mathrm{Pt} / \mathrm{NiO}(1) / \mathrm{YIG}$. The blocking temperatures are approximately determined to be around 70,110 , and $150 \mathrm{~K}$ for the $\mathrm{Pt} / \mathrm{NiO} / \mathrm{YIG}$ heterostructures with 1,3 , and $5 \mathrm{~nm} \mathrm{NiO}$ inserted layers, respectively. The coercivity $H_{\mathrm{c}}$ exhibits a sudden increase near $T_{b}$, as shown in the inset of Fig. 4(b).
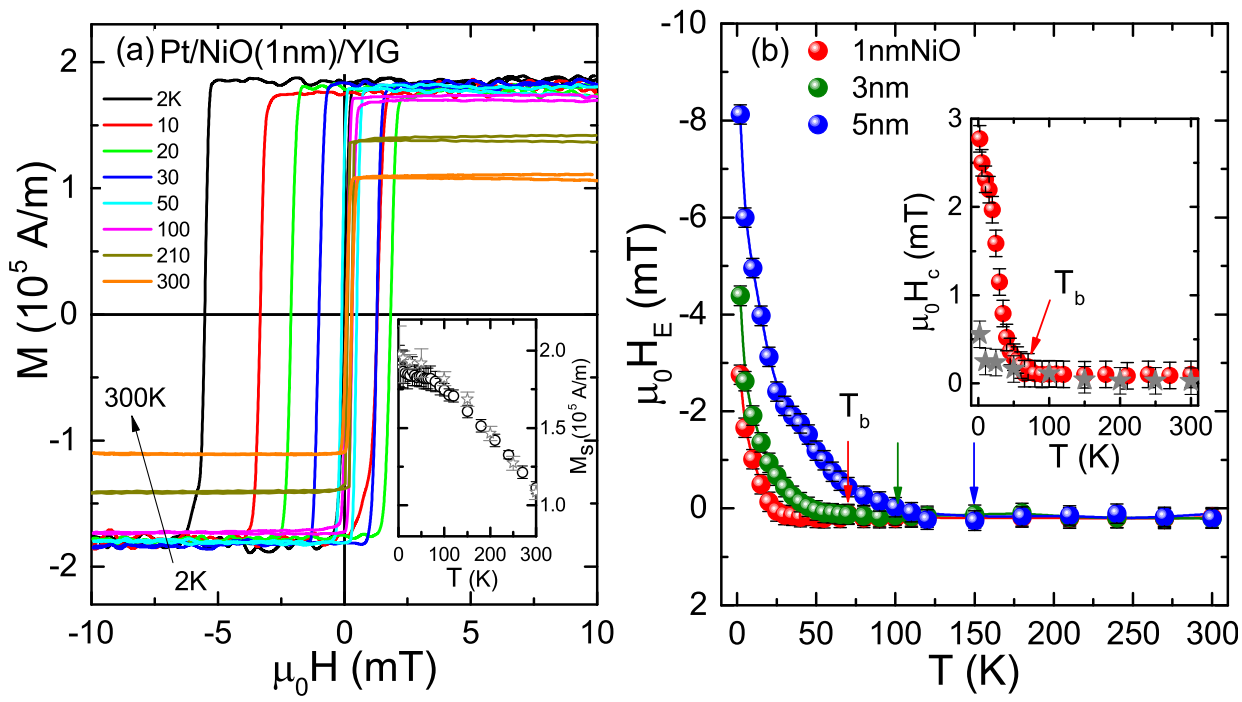

FIG. 4. (a) Hysteresis loops for Pt/ $\mathrm{NiO}(1) / \mathrm{YIG}$ at various temperatures. The inset shows the saturation magnetization $M_{\mathrm{s}}$ versus temperature for Pt/YIG (stars) and $\mathrm{Pt} / \mathrm{NiO}(1) / \mathrm{YIG}$ (circles). (b) Temperature dependent $H_{\mathrm{e}}$ for $\mathrm{Pt} / \mathrm{NiO} /$ YIG with different $\mathrm{NiO}$ thicknesses. The arrows indicate the blocking temperatures $T_{\mathrm{b}}$. The inset of (b) plots the temperature dependent coercivity $H_{\mathrm{c}}$ for $\mathrm{Pt} /$ YIG (stars) and Pt/NiO(1)/YIG (circles). 
While for $\mathrm{Pt} / \mathrm{YIG}$ films, $H_{\mathrm{c}}$ is rather small for the temperature range from $300 \mathrm{~K}(<0.1 \mathrm{mT})$ to $2 \mathrm{~K}(0.55 \mathrm{mT})$. The $\mathrm{Pt} / \mathrm{YIG}$ and $\mathrm{Pt} / \mathrm{NiO}(1) / \mathrm{YIG}$ films have identical temperature dependent saturation magnetization $M_{\mathrm{s}}$ as shown in the inset of Fig. 4(a), consistent with the previous reported values. ${ }^{28,33}$

We finally comment on the difference on the AFM mediated spin transport between the spin pumping or spin Seebeck result and our SMR result. In the former cases, the spin current was created in the YIG layer and the spin current detected in the $\mathrm{Pt}$ was found to be enhanced in the presence of the thin AFMI layer, and furthermore the spin current could penetrate to the AFMI as large as $10 \mathrm{~nm} .{ }^{18-22}$ In our case, both spin current creation and detection occur in the Pt layer, and we do not find any enhancement compared to the spin current without the AFMI layer, and the SMR disappears when the thickness of the AFMI exceeds $3 \mathrm{~nm}$. On the other hand, there is one common feature: all experiments have shown that the spin current transmission is strongest near or above the ordering temperature of the AFMI. Clearly, magnetic fluctuation is important in all these experiments; however, the different amplitude and thickness dependence of the spin signals in spin pumping or spin Seebeck compared with our data demand further theoretical and experiments on the spin transport physics in AFMIs.

In summary, we investigated the $\mathrm{SMR}$ in $\mathrm{Pt} / \mathrm{NiO} / \mathrm{YIG}$ heterostructures over a wide temperature range. The SMR signal is comparable to that without the NiO layer when the temperature is near or above the blocking temperature of the $\mathrm{NiO}$, but significantly suppressed at low temperatures. On the other hand, the CAMR is practically zero, implying that the insertion of the $\mathrm{NiO}$ has completely destroyed the $\mathrm{Pt}$ magnetization induced by the YIG MPE. The dual roles of the thin $\mathrm{NiO}$ layer are to suppress the magnetic interaction or MPE between Pt and YIG, and to maintain efficient spin current transmission at high temperature.

We thank the high magnetic field laboratory of Chinese Academy of Sciences for the FMR measurements. This work was financially supported by the National Natural Science foundation of China (Grant Nos. 11274321, 11404349, 51522105, 11374312, and 51502314) and the Key Research Program of the Chinese Academy of Sciences (Grant No. KJZD-EW-M05). S. Zhang was partially supported by the U. S. National Science Foundation (Grant No. ECCS1404542).

${ }^{1}$ S. Maekwa, Spin Current (Oxford University Press, Oxford, 2012).

${ }^{2}$ M. Z. Wu and A. Hoffmann, Recent Advances in Magnetic InsulatorsFrom Spintronics to Microwave Applications (Academic Press, San Diego, 2013), Vol. 64.

${ }^{3}$ B. Heinrich, C. Burrowes, E. Montoya, B. Kardasz, E. Girt, Young-Yeal Song, Y. Sun, and M. Z. Wu, Phys. Rev. Lett. 107, 066604 (2011).

${ }^{4}$ S. M. Rezende, R. L. Rodríguez-Suárez, M. M. Soares, L. H. Vilela-Leao, D. Ley Domínguez, and A. Azevedo, Appl. Phys. Lett. 102, 012402 (2013).
${ }^{5}$ Y. Kajiwara, K. Harii, S. Takahashi, J. Ohe, K. Uchida, M. Mizuguchi, H. Umezawa, H. Kawai, K. Ando, K. Takanashi, S. Maekawa, and E. Saitoh, Nature 464, 262 (2010).

${ }^{6}$ K. Uchida, S. Takahashi, K. Harii, J. Ieda, W. Koshibae, K. Ando, S. Maekawa, and E. Saitoh, Nature 455, 778 (2008).

${ }^{7}$ K. Uchida, J. Xiao, H. Adachi, J. Ohe, S. Takahashi, J. Ieda, T. Ota, Y. Kajiwara, H. Umezawa, H. Kawai, G. E. W. Bauer, S. Maekawa, and E. Saitoh, Nat. Mater. 9, 894 (2010).

${ }^{8}$ G. E. W. Bauer, E. Saitoh, and B. J. van Wees, Nat. Mater. 11, 391 (2012). ${ }^{9}$ J. E. Hirsch, Phys. Rev. Lett. 83, 1834 (1999).

${ }^{10}$ J. Wunderlich, B. Kaestner, J. Sinova, and T. Jungwirth, Phys. Rev. Lett. 94, 047204 (2005).

${ }^{11}$ Y. K. Kato, R. C. Myers, A. C. Gossard, and D. D. Awschalom, Science 306, 1910 (2004).

${ }^{12}$ J. B. S. Mendes, R. O. Cunha, O. Alves Santos, P. R. T. Ribeiro, F. L. A. Machado, R. L. Rodríguez-Suárez, A. Azevedo, and S. M. Rezende, Phys. Rev B 89, 140406(R) (2014).

${ }^{13}$ W. Zhang, M. B. Jungfleisch, W. Jiang, J. E. Pearson, A. Hoffmann, F. Freimuth, and Y. Mokrousov, Phys. Rev. Lett. 113, 196602 (2014).

${ }^{14}$ L. Frangou, S. Oyarzún, S. Auffret, L. Vila, S. Gambarelli, and V. Baltz, Phys. Rev. Lett. 116, 077203 (2016).

${ }^{15}$ J. Sinova, S. O. Valenzuela, J. Wunderlich, C. H. Back, and T. Jungwirth, Rev. Mod. Phys. 87, 1213 (2015) and reference therein.

${ }^{16}$ X. Zhou, L. Ma, Z. Shi, W. J. Fan, J. G. Zheng, R. F. L. Evans, and S. M. Zhou, Phys. Rev. B 92, 060402(R) (2015).

${ }^{17}$ S. Seki, T. Ideue, M. Kubota, Y. Kozuka, R. Takagi, M. Nakamura, Y. Kaneko, M. Kawasaki, and Y. Tokura, Phys. Rev. Lett. 115, 266601 (2015).

${ }^{18}$ C. Hahn, G. de Loubens, O. Klein, M. Viret, V. V. Naletov, and J. B. Youssef, Europhys. Lett. 108, 57005 (2014).

${ }^{19}$ H. L. Wang, C. H. Du, P. C. Hammel, and F. Y. Yang, Phys. Rev. Lett. 113, 097202 (2014).

${ }^{20}$ H. L. Wang, C. H. Du, P. C. Hammel, and F. Y. Yang, Phys. Rev. B 91, 220410(R) (2015)

${ }^{21}$ Z. Qiu, J. Li, D. Hou, E. Arenholz, A. T. NDiaye, A. Tan, K. Uchida, K. Sato, Y. Tserkovnyak, Z. Q. Qiu, and E. Saitoh, e-print arXiv:1505.03926.

${ }^{22}$ W. Lin, K. Chen, S. Zhang, and C. L. Chien, Phys. Rev. Lett. 116, 186601 (2016).

${ }^{23}$ R. Cheng, J. Xiao, Q. Niu, and A. Brataas, Phys. Rev. Lett. 113, 057601 (2014).

${ }^{24}$ S. Takei, T. Moriyama, T. Ono, and Y. Tserkovnyak, Phys. Rev. B 92, 020409(R) (2015).

${ }^{25}$ R. Khymyn, I. Lisenkov, V. S. Tiberkevich, A. N. Slavin, and B. A. Ivanov, Phys. Rev. B 93, 224421 (2016).

${ }^{26}$ S. M. Rezende, R. L. Rodríguez-Suárez, and A. Azevedo, Phys. Rev. B 93, 014425 (2016).

${ }^{27}$ A. Baruth and S. Adenwalla, Phys. Rev. B 78, 174407 (2008).

${ }^{28}$ M. C. Onbasli, A. Kehlberger, D. H. Kim, G. Jakob, M. Kläui, A. V. Chumak, B. Hillebrands, and C. A. Ross, APL Mater. 2, 106102 (2014) and reference therein.

${ }^{29}$ Y. T. Chen, S. Takahashi, H. Nakayama, M. Althammer, S. T. B. Goennenwein, E. Saitoh, and G. E. W. Bauer, J. Phys. Condens. Matter 28, 103004 (2016) and reference therein.

${ }^{30}$ T. Shang, H. L. Yang, Q. F. Zhan, Z. H. Zuo, Y. L. Xie, L. P. Liu, S. L. Zhang, Y. Zhang, H. H. Li, B. M. Wang, Y. H. Wu, S. Zhang, and R.-W. Li, e-print arXiv:1603.03578.

${ }^{31}$ M. Althammer, S. Meyer, H. Nakayama, M. Schreier, S. Altmannshofer, M. Weiler, H. Huebl, S. Geprägs, M. Opel, R. Gross, D. Meier, C. Klewe, T. Kuschel, J. M. Schmalhorst, G. Reiss, L. M. Shen, A. Gupta, Y. T. Chen, G. E. W. Bauer, E. Saitoh, and S. T. B. Goennenwein, Phys. Rev. B 87, 224401 (2013).

${ }^{32}$ T. Shang, Q. F. Zhan, H. L. Yang, Z. H. Zuo, Y. L. Xie, H. H. Li, L. P. Liu, B. M. Wang, Y. H. Wu, S. Zhang, and R.-W. Li, Sci. Rep. 5, 17734 (2015).

${ }^{33}$ P. Jang, S. Yamamoto, and H. Kuniki, Phys. Status Solidi (a) 201, 1851 (2004). 